ThE ASTrophysiCal Journal, 331:986-998, 1988 August 15

(C) 1988. The American Astronomical Society. All rights reserved. Printed in U.S.A.

\title{
UNRESOLVED VELOCITY STRUCTURE IN DIFFUSE INTERSTELLAR CLOUDS
}

\author{
JOHN H. BLACK \\ Steward Observatory \\ AND \\ EWINE F. VAN DisHOECK \\ Princeton University Observatory \\ Received 1987 December 22; accepted 1988 February 9
}

\begin{abstract}
Recent high-resolution $\left(\lambda / \Delta \lambda \approx 10^{5}\right)$ observations of interstellar absorption lines of $\mathrm{CH}$ and $\mathrm{CN}$ toward $\zeta$ Oph obtained by Crane et al. and Palazzi, Mandolesi, and Crane exhibit line widths that suggest thermal line broadening at high temperature, $T \approx 1200 \mathrm{~K}$. Observations of CO line emission at $\lambda=2.6 \mathrm{~mm}$ toward $\zeta$ Oph (Langer, Glassgold, and Wilson) indicate that the molecular gas resides in four distinct velocity components that span less than $3 \mathrm{~km} \mathrm{~s}^{-1}$ in Doppler velocity. Simulated $\mathrm{CH}$ and $\mathrm{CN}$ absorption line profiles are compared for high-temperature $(T=1200 \mathrm{~K})$ thermal broadening and for a combination of low-temperature $(T=50 \mathrm{~K})$ thermal plus turbulent $\left(\Delta V_{t}=0.4 \mathrm{~km} \mathrm{~s}^{-1}\right)$ broadening. It is shown that the two broadening models reproduce existing observations comparably well and are virtually indistinguishable at $\lambda / \Delta \lambda \approx 10^{5}$. The observed differences in the $\mathrm{CH}$ and $\mathrm{CN}$ line widths may reflect slightly different distributions of those molecules along the line of sight. The simulations use very recent, improved laboratory spectroscopic data on $\mathrm{CH}$ (Bernath). Some related consequences of such unresolved velocity structure on the ultraviolet absorption lines of $\mathrm{CO}$ are examined. Indirect diagnostics of temperature in the $\zeta$ Oph cloud favor low-temperature thermal plus turbulent broadening and the implied rate of dissipation of turbulence is in harmony with estimates of the global input of mechanical energy into the interstellar medium. Future needs for high-resolution spectroscopy are discussed.
\end{abstract}

Subject headings: interstellar: matter — interstellar: molecules - line formation — line profiles stars: individual $(\zeta \mathrm{Oph})$

\section{INTRODUCTION}

It has been recognized for some time that unresolved velocity structure in interstellar absorption lines at the level of a few kilometers per second or less can affect the determination of column densities and abundances from integrated line intensities. For example, Nachman and Hobbs (1973) discussed the effect of multiple components on the application of the "doublet-ratio" method for locating equivalent widths on a curve of growth. Crutcher (1975) considered the velocity structure along the line of sight to $\zeta$ Oph and subsequently (Crutcher 1977) used complex velocity distributions in a reanalysis of heavy-element abundances toward $\zeta \mathrm{Oph}$ and $\sigma$ Sco. In a reinterpretation of Copernicus ultraviolet spectra of interstellar O I, de Boer $(1979,1981)$ accounted for the effects of multiple velocity components through the use of visible absorption line spectra of other atoms obtained at higher resolution (e.g., Hobbs 1973). Snow and Meyers (1979) also redetermined column densities of several species in the complex interstellar line system of $\zeta O \mathrm{Oph}$.

There are several types of remedies for the afflictions due to unresolved structure. One is simply to perform measurements at higher resolution. Another is to use higher resolution data on profiles of other species that are assumed to coexist with species of interest in order to interpret less well-resolved lines. Unresolved structure in profiles affects abundance determinations when corrections for saturation (curve-of-growth effects) are appreciable. Abundance determinations can be improved through measurement of intrinsically weak transitions that are unsaturated or only slightly so. For many pur- poses, empirical curves of growth can be tolerated as useful substitutes for detailed knowledge of line profiles and saturation effects (Jenkins 1986).

All of the above studies at ultraviolet and visible wavelengths were concerned with velocity structure at the level of a few $\mathrm{km} \mathrm{s}^{-1}$. For example, Crutcher (1975) referred to five distinct components toward $\zeta$ Oph with LSR radial velocities $V_{\mathrm{LSR}}=-13.3,-2.8,-0.3,+1.8$, and $+4.5 \mathrm{~km} \mathrm{~s}^{-1}$, where most of the neutral atomic and molecular material is located in the $-0.3 \mathrm{~km} \mathrm{~s}^{-1}$ component. Until recently, there has not been a recognized and compelling need for information with resolution better than $1 \mathrm{~km} \mathrm{~s}^{-1}$. Resolving powers of $\lambda / \Delta \lambda=10^{4}-10^{5}$, corresponding to velocity resolutions of $\Delta V=c \Delta \lambda / \lambda \approx 3-30 \mathrm{~km} \mathrm{~s}^{-1}$, have been used for interstellar absorption line observations in the near-infrared, visible, and ultraviolet. There exists a body of visible absorption line data at $\Delta V \approx 1 \mathrm{~km} \mathrm{~s}^{-1}$ (Hobbs 1978a, b, and references therein). Some measurements have achieved sufficiently high resolution and signal/noise ratio on relatively weak lines that hyperfine structure in the interstellar $\mathrm{Na} \mathrm{D}$ lines can be discerned (Wayte, Wynne-Jones, and Blades 1978; Blades, Wynne-Jones, and Wayte 1980), and Doppler parameters as small as $b \approx 0.3$ $\mathrm{km} \mathrm{s}^{-1}$ can be found. The data of highest resolution are of minor atomic species and one molecule, $\mathrm{CH}^{+}$, for which there is good reason to expect atypical profiles and velocity distributions. Detailed studies of profiles of molecular absorption lines are lacking.

Very recently, Crane et al. (1986) and Palazzi, Mandolesi, and Crane (1988) have obtained observations of the $\mathrm{CH}$ and 
$\mathrm{CN}$ lines at $\lambda / \Delta \lambda \approx 10^{5}$ toward $\zeta$ Oph that permit detailed comparison of line widths, although not of resolved profiles. Palazzi, Mandolesi, and Crane conclude from this comparison that the line widths of $\mathrm{CH}$ and $\mathrm{CN}$, after correction for instrumental broadening, are in exactly the ratio expected for thermal Doppler broadening at high temperature $(T \approx 1200 \mathrm{~K})$ with no measurable turbulent contribution. The observational result itself is compelling, and its interpretation in terms of high-temperature molecular gas contrasts sharply with diagnostic data on other molecules in the same absorbing region that suggest a much lower kinetic temperature, $T \approx 30-100 \mathrm{~K}$ (van Dishoeck and Black 1986a, b). The alternative possibility is that a complicated distribution in velocity of cold absorbing gas along the line of sight causes the $\mathrm{CH}$ and $\mathrm{CN}$ lines to be broadened in just such a way as to mimic high-temperature thermal broadening. In the absence of any evidence for velocity structure not revealed at $\lambda / \Delta \lambda \approx 10^{5}$, this would be a completely ad hoc explanation of no great value. However, Langer, Glassgold, and Wilson (1987) have recently identified four distinct components at LSR radial velocities of $V_{\mathrm{LSR}}=-2.0$, $-0.72,0.00$, and $+0.64 \mathrm{~km} \mathrm{~s}^{-1}$ in the $\lambda=2.6 \mathrm{~mm} \mathrm{CO}$ emission lines in the direction of $\zeta \mathrm{Oph}$ with line widths less than $0.5 \mathrm{~km} \mathrm{~s}^{-1}$. These data have been obtained at resolving powers of $\lambda / \Delta \lambda=4.6 \times 10^{6}$ and $2.3 \times 10^{6}$, corresponding to velocity resolutions of $\Delta V=0.065$ and $0.13 \mathrm{~km} \mathrm{~s}^{-1}$, respectively. The mean velocity of these components weighted by the column density of $\mathrm{CO}$ in each is $V_{\mathrm{LSR}}=-0.31 \mathrm{~km} \mathrm{~s}^{-1}$, which is in excellent agreement with the velocity of the principal optical absorption features observed at much lower resolution. Moreover, the total extent in velocity of the $\mathrm{CO}$ emission is comparable to the resolution of the $\mathrm{CH}$ and $\mathrm{CN}$ measurements. Finally, the good agreement between the CO column density inferred from ultraviolet absorption (Wannier, Penzias, and Jenkins 1982) and millimeter emission observations (Langer et al. 1987) strongly suggests that all CO seen at millimeter wavelengths is located in front of $\zeta \mathrm{Oph}$. Thus the velocity distribution observed by Langer, Glassgold, and Wilson can be used to test the interpretation of the $\mathrm{CH}$ and $\mathrm{CN}$ line broadening, subject only to the assumption that the $\mathrm{CH}$ and $\mathrm{CN}$ are distributed among the velocity components proportionally to the CO. The similarity of $\mathrm{CH} 9 \mathrm{~cm}$ and $\mathrm{CO} 2.6 \mathrm{~mm}$ emission line profiles measured by Liszt (1979) supports this assumption, even though these observations were performed with rather large beam sizes.

In the following, we present a detailed comparison of simulated absorption line profiles that represent simple, hightemperature thermal broadening and low-temperature thermal plus turbulent broadening with complex, unresolved velocity structure. We consider other effects of unresolved structure on the scale of $0.1-1.0 \mathrm{~km} \mathrm{~s}^{-1}$, including the problems associated with determining the $\mathrm{CO}$ abundance and the implications of small isotope shifts and hyperfine structure. It is evident that further efforts are urgently needed in the high-resolution spectroscopy of interstellar clouds to elucidate their physical conditions and internal structure, as well as to provide accurate abundance determinations. It is also clear that resolving powers of $\lambda / \Delta \lambda \approx 10^{5}$ are not at all high enough for some purposes.

\section{LINE PROFILES}

We are concerned here with the interpretation of absorption line observations in terms of theoretical profiles that simulate both the instrumental response and the physical conditions in the absorbing gas. The net profile function

$$
\phi(\lambda)=\int \exp \left[-\tau\left(\lambda^{\prime}\right)\right] g\left(\lambda-\lambda^{\prime}\right) d \lambda^{\prime},
$$

is the convolution of a true, pure absorption profile specified by an optical depth function $\tau(\lambda)$ and an instrumental function $g\left(\lambda-\lambda^{\prime}\right)$. In the present context, stimulated emission can be ignored; therefore,

$$
\tau(\lambda)=2.4932 \times 10^{-15} \sum_{j} f_{j} N_{j} \lambda_{j} \mathscr{V}\left(x_{j}, y_{j}\right) / \Delta V_{j}
$$

where each line component $j$ contributing to the absorption is described by a lower state column density $N_{j}\left(\mathrm{~cm}^{-2}\right)$, an absorption oscillator strength $f_{j}$, a line center wavelength $\lambda_{j}$ $(\AA)$, and a true width $\Delta V_{j}\left(\mathrm{~km} \mathrm{~s}^{-1}\right)$. The inherent profile is given by a Voigt function

$$
\mathscr{V}\left(x_{j}, y_{j}\right)=\frac{y_{j}}{\pi} \int_{-\infty}^{+\infty} \frac{\exp \left(-t^{2}\right)}{y_{j}^{2}+\left(x_{j}-t\right)^{2}} d t
$$

where

$$
y_{j}=1.32505 \times 10^{-14} A_{j} \lambda_{j} / \Delta V_{j}
$$

is the ratio of natural and Doppler widths in terms of the inverse lifetime, $A_{j}\left(\mathrm{~s}^{-1}\right)$, of the upper state of transition $j$, and

$$
x_{j}=4.99187 \times 10^{5}\left(\frac{\lambda_{j}}{\lambda}-1\right) / \Delta V_{j}
$$

is the separation from line center measured in Doppler halfwidths. For unresolved lines, the principal observable property is not the profile itself, but the equivalent width,

$$
W_{\lambda}=\int[1-\phi(\lambda)] d \lambda .
$$

Throughout this discussion the line widths of individual components are characterized by the full width at half-peak of the Doppler (Gaussian) core, $\Delta \lambda$ in wavelength units or $\Delta V=$ $c \Delta \lambda / \lambda_{0}$ in corresponding Doppler velocity units. The conventional Doppler parameter $b$ is related to this width by $\Delta V=2(\ln 2)^{1 / 2} b=1.665 b$. For purely thermal Doppler broadening, the width is determined by the Doppler temperature $T_{\mathrm{D}}(\mathrm{K})$ and the mass of the absorbing atom or molecule, $M(\mathrm{amu})$,

$$
\Delta V=0.21472\left(T_{\mathrm{D}} / M\right)^{1 / 2} \mathrm{~km} \mathrm{~s}^{-1}
$$

The instrumental function is taken to be a Gaussian

$$
g(z)=0.93944 \exp \left(-2.77259 z^{2}\right) / \Delta \lambda_{i}
$$

where $\Delta \lambda_{i}$ is the full width at half-peak of the instrumental function in wavelength units, and $z=\left(\lambda-\lambda_{0}\right) / \Delta \lambda_{i}$. If both the true and instrumental profiles are Gaussians, then their widths combine in quadrature to give the apparent width of each component

$$
\Delta \lambda=\left[\left(\Delta \lambda_{j}\right)^{2}+\left(\Delta \lambda_{i}\right)^{2}\right]^{1 / 2}
$$

$$
\text { III. INTERSTELLAR CH AND CN }
$$

The recent work of Crane et al. (1986) and Palazzi, Mandolesi, and Crane (1988) provides extremely accurate information on apparent line widths and equivalent widths of blue-violet absorption lines of interstellar $\mathrm{CH}$ (3878 and $3886 \AA)$ and $\mathrm{CN}$ $(3874 \AA)$ toward $\zeta$ Oph. The maximum resolving power of 
these observations, $\lambda / \Delta \lambda=156,000$, corresponds to a velocity resolution $\Delta V_{i}=1.92 \mathrm{~km} \mathrm{~s}^{-1}$ that is still large enough to dominate the apparent broadening of narrow interstellar lines such as these. After correcting the apparent widths for instrumental broadening (as in eq. [9]), Palazzi, Mandolesi, and Crane (1988) found a ratio of true widths, $\Delta V_{\mathrm{CH}} / \Delta V_{\mathrm{CN}}=1.44 \pm 0.04$. For comparison, the ratio expected for pure thermal broadening (eq. [7]) is $\Delta V_{\mathrm{CH}} / \Delta V_{\mathrm{CN}}=1.414$, if $\mathrm{CH}$ and $\mathrm{CN}$ exist in the same region. Thus the line width measurements are in harmony with pure thermal broadening and the implied temperature is $T_{\mathrm{D}} \approx 1200 \mathrm{~K}$ (Palazzi et al. 1988). The unresolved splitting of the two lines that make up the $3886 \AA$ feature cannot account for the entire effect of broadening because the unblended $3878 \AA$ line of $\mathrm{CH}$ is measured to have the same width. The only alternative explanation seems to be that a complex velocity distribution of cold absorbers mimics in detail the effects of high-temperature thermal broadening.

Results are presented for two models of the line broadening. Model 1 is a representation of the thermal broadening interpretation of Palazzi et al. In this case it is assumed that $T_{\mathrm{D}}=$ $1200 \mathrm{~K}$; therefore, $b=0.876 \mathrm{~km} \mathrm{~s}^{-1}$ for $\mathrm{CN}$ and $b=1.238 \mathrm{~km}$ $\mathrm{s}^{-1}$ for $\mathrm{CH}$. In model 2 , we initially assume that the $\mathrm{CH}$ and $\mathrm{CN}$ are distributed in the same way as the $\mathrm{CO}$ molecules in four distinct velocity components. Accordingly, we adopt for $\mathrm{CH}$ and $\mathrm{CN}$ the radial velocities $V_{j}$ and relative column densities $N_{j} / N$ as measured for these four components for $\mathrm{CO}$ by Langer, Glassgold and Wilson (1987). The broadening of each velocity component is taken to have a thermal Doppler part at $T=T_{\mathrm{D}}=50 \mathrm{~K}$ and a turbulent contribution characterized by a Gaussian width $\Delta V_{t}=0.4095 \mathrm{~km} \mathrm{~s}^{-1}$ such that the apparent width

$$
(\Delta V)^{2}=\left(\Delta V_{i}\right)^{2}+\left(\Delta V_{t}\right)^{2}+8(\ln 2) k T / M .
$$

These parameters are in harmony with the inherent $\mathrm{CO}$ line widths $\Delta V_{\mathrm{CO}}=0.5 \mathrm{~km} \mathrm{~s}^{-1}$ inferred for each component by Langer, Glassgold, and Wilson. Model 3 of the line broadening is a variant of model 2 in which the $\mathrm{CH}$ is assumed to be distributed in slightly different proportions among the velocity components than the $\mathrm{CN}$ and $\mathrm{CO}$.
TABLE 1

$\zeta$ Oph LiNe Broadening Models

\begin{tabular}{cccc}
\hline \hline Property & Model 1 & Model 2 & Model 3 \\
\hline$N(\mathrm{CN})\left(\mathrm{cm}^{-2}\right) \ldots \ldots \ldots$ & $2.86(12)$ & $2.955(12)$ & $2.955(12)$ \\
$b(\mathrm{CN})\left(\mathrm{km} \mathrm{s}^{-1}\right) \ldots \ldots \ldots$ & 0.876 & 0.304 & 0.304 \\
$T_{\mathrm{e}}(\mathrm{CN})(\mathrm{K}) \ldots \ldots \ldots \ldots$ & 2.80 & 2.80 & 2.80 \\
$N(\mathrm{CH})\left(\mathrm{cm}^{-2}\right) \ldots \ldots \ldots$ & $2.50(13)$ & $2.550(13)$ & $2.52(13)$ \\
$b(\mathrm{CH})\left(\mathrm{km} \mathrm{s}^{-1}\right) \ldots \ldots$. & 1.238 & 0.353 & 0.353 \\
$T(\mathrm{~K}) \ldots \ldots \ldots \ldots \ldots$. & 1200. & 50. & 50. \\
$\Delta V_{t}\left(\mathrm{~km} \mathrm{~s}^{-1}\right) \ldots \ldots \ldots$. & 0.0 & 0.4095 & 0.4095 \\
$V_{j}, N_{j} / N \ldots \ldots \ldots \ldots \ldots$ & $0.0,1.00$ & $-2.00,0.02$ & $-2.00,0.15$ \\
& $\ldots$ & $-0.72,0.58$ & $-0.72,0.50$ \\
& $\ldots$ & $0.00,0.17$ & $0.00,0.15$ \\
& $\ldots$ & $+0.64,0.23$ & $+0.64,0.20$ \\
\hline
\end{tabular}

The three line broadening models are summarized in Table 1. All models adopt a uniform rotational excitation temperature $T_{\mathrm{ex}}=2.80 \mathrm{~K}$ for $\mathrm{CN}$, as found by Crane et al. (1986). In all cases, the two $\Lambda$-doubling levels of the $\mathrm{CH} X{ }^{2} \Pi_{1 / 2}$ $J=1 / 2$ state are taken to be equally populated (Palazzi et al. 1988; Jura and Meyer 1985). Given the adopted line broadening parameters, the tabulated column densities are the values that provide the best fits to the observations. The best-fitting column densities of the three models agree within $3.3 \%$. The differences are small because the lines are only weakly saturated.

The adopted rest wavelengths and oscillator strengths are given in Table 2. The fractional population in the initial (lower) state of each line is $\eta$. As pointed out previously, the published line positions for the $\mathrm{CH} B-X(0,0)$ system are poorly determined in relation to current astrophysical requirements (Danks, Federman, and Lambert 1984; Palazzi et al. 1988). The positions of all lines in this system are expected to be uncertain by at least $0.5-1 \mathrm{~km} \mathrm{~s}^{-1}$, based on the measurements of Gerö (1941) and their reanalysis by Botterud, Lofthus, and Veseth (1973). The low- $J$ lines of the $(0,0)$ band of the $B-X$ system have recently been remeasured more accurately by Bernath (1987b). Positions of the interstellar lines based upon Bernath's measurements and best-fitting spectroscopic con-

TABLE 2

Adopted Line Parameters for CH ANd CN

\begin{tabular}{|c|c|c|c|c|c|c|}
\hline Transition & $\begin{array}{c}(v / \mathrm{c})_{\text {vacuum }} \\
\left(\mathrm{cm}^{-1}\right)\end{array}$ & $\begin{array}{l}\lambda_{\text {air }} \\
(\AA)\end{array}$ & $f$ & $\begin{array}{c}A \\
\left(\mathrm{~s}^{-1}\right)\end{array}$ & $\eta$ & Notes \\
\hline \multicolumn{7}{|l|}{ CH $B^{2} \Sigma^{-}-X^{2} \Pi(0,0):$} \\
\hline${ }^{P} Q_{12}(1 / 2)$ & 25698.2250 & 3890.2170 & $2.13(-3)$ & $3.05(6)$ & 0.5 & \multirow[t]{4}{*}{1,2} \\
\hline$R_{2}(1 / 2) \ldots$ & 25774.0368 & 3878.7740 & $1.07(-3)$ & $3.05(6)$ & 0.5 & \\
\hline${ }^{Q} R_{12}(1 / 2)$ & 25723.3762 & 3886.4132 & $1.07(-3)$ & $3.05(6)$ & 0.5 & \\
\hline$Q_{2}(1 / 2) \ldots \ldots \ldots \ldots \ldots \ldots \ldots \ldots \ldots \ldots \ldots \ldots \ldots$ & 25723.4181 & 3886.4069 & $2.13(-3)$ & $3.05(6)$ & 0.5 & \\
\hline \multicolumn{7}{|l|}{$\mathrm{CH} A^{2} \Delta-X^{2} \Pi(0,0):$} \\
\hline$R_{\mathrm{fe}}(1 / 2) \ldots$ & 23247.6357 & 4300.3030 & $5.06(-3)$ & $1.866(6)$ & 0.5 & \multirow[t]{2}{*}{3,4} \\
\hline$R_{\mathrm{ff}}(1 / 2) \quad \ldots \ldots \ldots \ldots \ldots$ & 23247.5249 & 4300.3235 & $5.06(-3)$ & $1.866(6)$ & 0.5 & \\
\hline \multicolumn{7}{|l|}{$\mathrm{CN} B^{2} \Sigma^{+}-X^{2} \Sigma^{+}(0,0):$} \\
\hline$R_{1}(2)+R_{2}(2)+{ }^{R} Q_{21}(2) \ldots \ldots \ldots \ldots$ & 25809.999 & 3873.370 & $2.03(-2)$ & $1.61(7)$ & $1.017(-2)$ & \multirow[t]{5}{*}{5,6} \\
\hline$R_{1}(1)+R_{2}(1)+{ }^{R} Q_{21}(1) \ldots$ & 25805.804 & 3873.999 & $2.25(-2)$ & $1.61(7)$ & $2.975(-1)$ & \\
\hline$R_{1}(0)+{ }^{R} Q_{21}(0) \ldots \ldots \ldots \ldots \ldots \ldots \ldots$ & 25801.754 & 3874.607 & $3.38(-2)$ & $1.61(7)$ & $6.924(-1)$ & \\
\hline${ }^{P} Q_{12}(1)+P_{1}(1) \ldots \ldots \ldots \ldots \ldots \ldots \ldots$ & 25794.052 & 3875.764 & $1.13(-2)$ & $1.61(7)$ & $2.975(-1)$ & \\
\hline${ }^{P} Q_{12}(2)+P_{1}(2)+P_{2}(2) \ldots \ldots \ldots \ldots$ & 25790.411 & 3876.312 & $1.35(-2)$ & $1.61(7)$ & $1.017(-2)$ & \\
\hline
\end{tabular}

NoTES. - (1) Line positions are based on measurements and preliminary analysis of Bernath $1987 b$; (2) oscillator strengths and lifetimes from Brzozowski et al. 1976; (3) line positions based on measurements of Brazier and Brown 1984 and X-state term values of Bernath $1987 a$; (4) oscillator strengths and lifetimes adapted from Larsson and Siegbahn 1983, but see text; (5) line positions from Engleman 1974; (6) oscillator strengths and lifetimes from Larsson, Siegbahn, and Ågren 1983. 
stants are listed in Table 2. The wavelength of the $R_{2}(1 / 2)$ line is $0.031 \AA$ smaller than the old value, which corresponds to a velocity difference of $-2.4 \mathrm{~km} \mathrm{~s}^{-1}$. The splitting of the $Q_{2}(1 / 2)+{ }^{Q} R_{12}(1 / 2)$ line pair is better determined than the absolute positions: $\Delta \lambda=6.33 \pm 0.04 \mathrm{~m} \AA$, or $\Delta V=$ $0.488 \pm 0.003 \mathrm{~km} \mathrm{~s}^{-1}$. This splitting is smaller than the value, $\Delta V=1.3 \mathrm{~km} \mathrm{~s}^{-1}$, inferred from the older spectroscopic data, and the difference can have a significant effect on discussions of interstellar line broadening. The weighted mean position of this unresolved line pair is $3886.4167 \AA$ in air.

For the $\mathrm{CH} A-X(0,0) R_{2}(1)=R_{f}(1 / 2)$ lines, we present in Table 2 improved wavelengths based on the ground-state term values of Bernath (1987a) and combination differences computed from measured line frequencies of Brazier and Brown (1984). The mean wavelength of the $A-X \quad \Lambda$-doublet, $4300.3132 \AA$ differs somewhat (by $0.54 \mathrm{~km} \mathrm{~s}^{-1}$ in Doppler velocity) from the previously accepted and less accurate value, 4300.321 § (Danks, Federman, and Lambert 1984). The CN "lines" are all unresolved blends and are assumed to be fundamentally unresolvable.

Theoretical line profiles have been computed as described in $\S$ II. An instrumental width $\Delta \lambda_{i}=24.8 \mathrm{~m} \AA$ has been adopted for the $\mathrm{CH} B-X$ and $\mathrm{CN} B-X$ line profiles for comparison with the observations. The spectrum in the region of the $\mathrm{CN}$ lines is shown in Figure $1 a$. The profiles for models 1 and 2 are virtually indistinguishable on the scale of this plot. Figure $1 b$ shows an expanded view of the $\mathrm{CN} B-X(0,0) R_{1}(0)$ profile. At this resolution there are slight differences in apparent widths and in central depths between models 1 and 2, but these are small in comparison with the observational uncertainties. The slight offset in effective central wavelengths results from the arbitrary identification of the $V_{\mathrm{LSR}}=0.0 \mathrm{~km} \mathrm{~s}^{-1}$ velocity component with the true rest wavelength in model 2 . The dotted curve in Figure $1 b$ is model 2 at a 5 times higher resolution of $4.96 \mathrm{~m} \AA$. Only at this high resolution is the velocity structure revealed and are the differences between models 1 and 2 apparent.

In Figures $2 a$ and $2 b$, detailed profiles of the $\mathrm{CH} B-X(0,0)$ $R_{2}(1 / 2)$ and $Q_{2}(1 / 2)+{ }^{Q} R_{12}(1 / 2)$ lines are presented. The pro- files obtained with model 2 are narrower and deeper than those of model 1, which match the observations well. Model 3 represents a relatively small modification of the distribution of molecules among the velocity components of model 2 and reproduces the observed line widths and depths quite well. The $\mathrm{CH}$ profiles of models 1 and 3 are virtually identical and the velocity structure becomes noticeable only at much higher resolution. The smaller intrinsic splitting of the $3886 \AA$ line pair causes this feature to be only slightly broader than the unblended $3878 \AA$ line in all models. The difference will become evident only at resolutions better than $1 \mathrm{~km} \mathrm{~s}^{-1}$.

The comparison of observable parameters is presented quantitatively for the best-fitting calculations at a resolution of $24.8 \mathrm{~m} \AA$ in Table 3 . The parameter $I_{0}$ is the central depth, relative to $I=1.0$ in the adjacent continuum, of the apparent profile. As is evident from Table 3 , all equivalent widths and line depths agree with the observed values within the quoted uncertainties. A few line widths have been determined for the calculated spectra in the same way as for observed spectra; that is, by least-squares fits of Gaussian functions to the theoretical spectra sampled at $0.5 \Delta \lambda_{i}$ intervals. The uncertainties listed for $\Delta \lambda$ of the calculated profiles are thus the fitting errors for these Gaussians. For the $\mathrm{CN}$ line widths, models 1 and 2 are in harmony. For $\mathrm{CH}$, models 1 and 3 yield almost equivalent results. It should be emphasized that while models 2 and 3 reproduce the integrated intensities and central depths of all the observed lines, they also account implicitly for the remarkably uniform excitation temperature of the $\mathrm{CN}$.

The $\mathrm{CH}$ lines of model 2 are not systematically broader than the $\mathrm{CN}$ lines, and it is necessary to modify the $\mathrm{CH}$ distribution in order to reproduce the observed widths. The comparison of results for models 2 and 3 shows that the profiles are quite sensitive to small differences in the distributions of $\mathrm{CH}$ and $\mathrm{CN}$ over the four components. Our theoretical cloud models (van Dishoeck and Black 1986b) indicate that CO and $\mathrm{CN}$ are found mostly in the densest parts of the cloud, whereas $\mathrm{CH}$ is distributed more evenly throughout. If the four components toward $\zeta$ Oph had different densities with, for example, the highest density occurring in the $-0.72 \mathrm{~km} \mathrm{~s}^{-1}$ component,

TABLE 3

Comparison of Observable Line Parameters

\begin{tabular}{|c|c|c|c|c|c|c|c|c|}
\hline $\begin{array}{l}\text { Molecule } \\
\text { Line }\end{array}$ & $\begin{array}{c}\mathrm{CN} \\
3873.4\end{array}$ & $\begin{array}{c}\mathrm{CN} \\
3874.0\end{array}$ & $\begin{array}{c}\mathrm{CN} \\
3874.6\end{array}$ & $\begin{array}{c}\mathrm{CN} \\
3875.8\end{array}$ & $\begin{array}{c}\text { CN } \\
3876.3\end{array}$ & $\begin{array}{c}\mathrm{CH} \\
3878.8\end{array}$ & $\underset{3886.4}{\mathrm{CH}}$ & $\underset{4300.3}{\text { CH }}$ \\
\hline $\begin{array}{l}\text { Observed }^{\mathrm{a}}: \\
\quad W_{\lambda}(\mathrm{m} \AA) \\
\Delta \hat{\lambda}(\mathrm{m} \AA) \\
I_{0} \quad \ldots \ldots \ldots \ldots \ldots \ldots \ldots\end{array}$ & $\begin{array}{c}0.072 \pm 0.026 \\
\ldots \\
0.99\end{array}$ & $\begin{array}{c}2.420 \pm 0.051 \\
\ldots \\
0.94\end{array}$ & $\begin{array}{c}7.646 \pm 0.091 \\
31.8 \pm 0.3 \\
0.79\end{array}$ & $\begin{array}{c}1.254 \pm 0.067 \\
\ldots \\
0.97\end{array}$ & $\begin{array}{l}\cdots \\
\cdots \\
\cdots\end{array}$ & $\begin{array}{c}1.74 \pm 0.02 \\
36.9 \pm 0.3 \\
0.96\end{array}$ & $\begin{array}{l}5.01 \pm 0.02 \\
36.2 \pm 0.3 \\
0.90\end{array}$ & $\begin{array}{c}17.8 \pm 0.9^{b} \\
\ldots \\
\cdots\end{array}$ \\
\hline $\begin{array}{l}\text { Model 1: } \\
\qquad W_{\lambda}(\mathrm{m} \AA) \\
\Delta \lambda(\mathrm{m} \AA) \\
I_{0} \quad \ldots \ldots \ldots \ldots \ldots \ldots \ldots\end{array}$ & $\begin{array}{c}0.0783 \\
\ldots \\
0.998\end{array}$ & $\begin{array}{c}2.433 \\
\ldots \\
0.927\end{array}$ & $\begin{array}{c}7.654 \\
31.6 \pm 0.3 \\
0.773\end{array}$ & $\begin{array}{l}1.250 \\
\ldots \\
0.963\end{array}$ & $\begin{array}{c}0.0522 \\
\ldots \\
0.998\end{array}$ & $\begin{array}{c}1.741 \\
35.8 \pm 1.8 \\
0.955\end{array}$ & $\begin{array}{l}5.022 \\
37.6 \pm 0.01 \\
0.874\end{array}$ & $\begin{array}{c}17.50 \\
\ldots \\
0.756\end{array}$ \\
\hline $\begin{array}{l}\text { Model 2: } \\
W_{\lambda}(\mathrm{m} \AA) \\
\Delta \lambda(\mathrm{d} \AA) \ldots \ldots \ldots \\
I_{0} \quad \ldots \ldots \ldots \ldots \ldots \ldots \ldots \\
\end{array}$ & $\begin{array}{l}0.0807 \\
\ldots \\
0.998\end{array}$ & $\begin{array}{c}2.481 \\
\ldots \\
0.928\end{array}$ & $\begin{array}{c}7.601 \\
32.4 \pm 0.4 \\
0.781\end{array}$ & $\begin{array}{c}1.283 \\
\ldots \\
0.963\end{array}$ & $\begin{array}{c}0.0539 \\
\ldots \\
0.998\end{array}$ & $\begin{array}{c}1.751 \\
31.8 \pm 1.5 \\
0.949\end{array}$ & $\begin{array}{c}4.996 \\
33.3 \pm 0.09 \\
0.859\end{array}$ & $\begin{array}{c}16.68 \\
\ldots \\
0.757\end{array}$ \\
\hline $\begin{array}{l}\text { Model 3: } \\
W_{\lambda}(\mathrm{m} \AA) \\
\Delta \lambda(\mathrm{m} \AA) \\
I_{0} \ldots \ldots \ldots \ldots \ldots \ldots \ldots\end{array}$ & $\begin{array}{c}0.0807 \\
\cdots \\
0.998\end{array}$ & $\begin{array}{c}2.481 \\
\ldots \\
0.928\end{array}$ & $\begin{array}{c}7.601 \\
32.4 \pm 0.4 \\
0.781\end{array}$ & $\begin{array}{c}1.283 \\
\ldots \\
0.963\end{array}$ & $\begin{array}{c}0.0539 \\
\ldots \\
0.998\end{array}$ & $\begin{array}{c}1.744 \\
35.6 \pm 1.7 \\
0.955\end{array}$ & $\begin{array}{l}5.028 \\
37.3 \pm 0.05 \\
0.873\end{array}$ & $\begin{array}{c}16.93 \\
\ldots \\
0.763\end{array}$ \\
\hline
\end{tabular}

a Observed values from Crane et al. 1986 and Palazzi et al. 1988 unless otherwise indicated

b Measurements at a resolving power $\lambda / \Delta \lambda=8 \times 10^{4}$ by Danks et al. 1984 . 

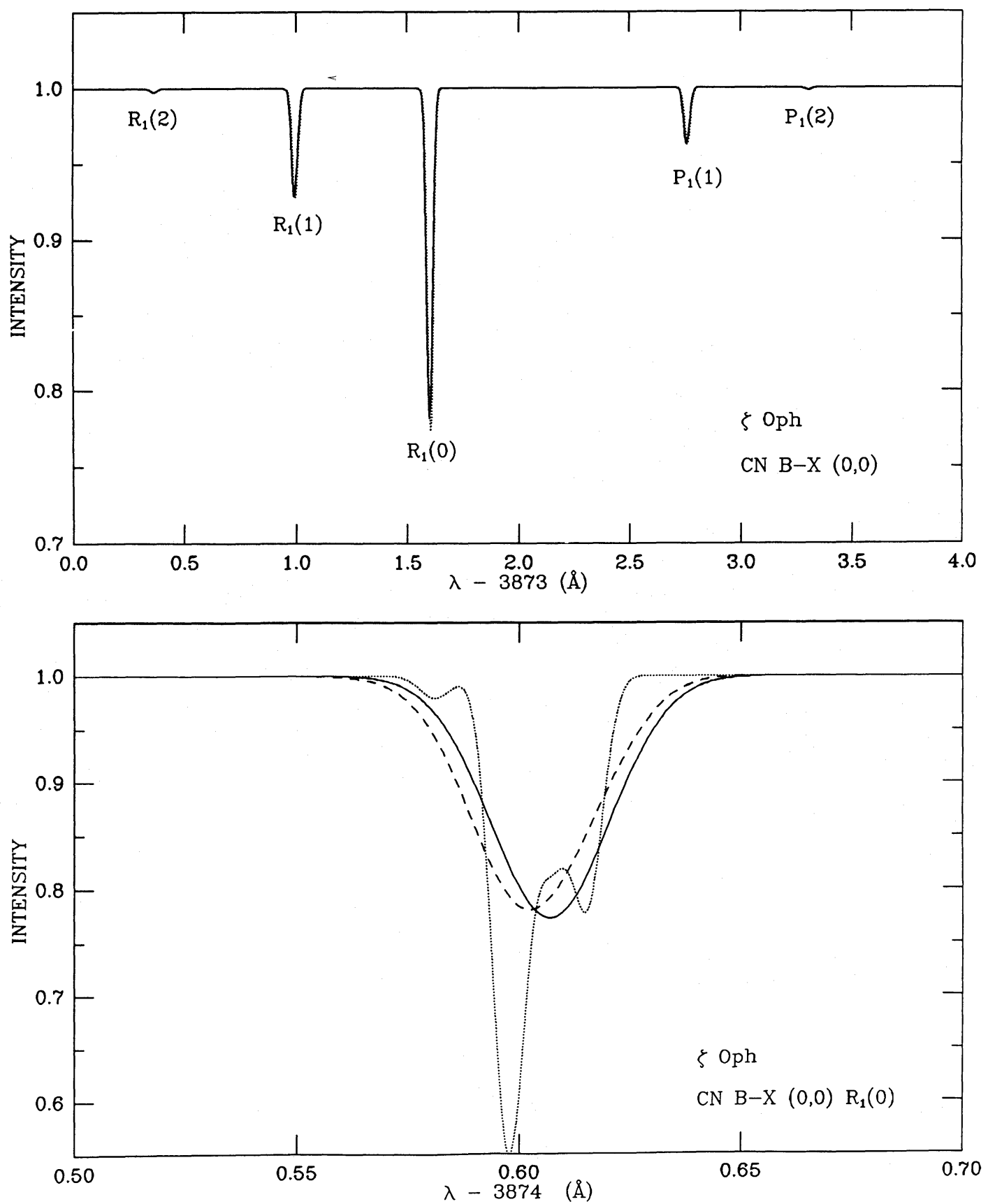

Fig. 1.-Top: theoretical spectra of interstellar CN $B-X(0,0)$ lines towards $\zeta$ Oph. Solid curve is the spectrum for model 2 . Dotted curve, which is barely distinguishable, is for model 1. Bottom: theoretical spectra of the CN $B-X(0,0) R(0)$ interstellar absorption feature toward $\zeta$ Oph. Solid curve (model 1$)$ is computed for a single velocity component with $b=0.876 \mathrm{~km} \mathrm{~s}^{-1}$. Dashed curve (model 2) is computed for four closely spaced velocity components, each of which has $b=0.304$ $\mathrm{km} \mathrm{s}^{-1}$. The resolution is $24.8 \mathrm{~m} \AA$ for both curves. Dotted curve is model 2 at a resolution of $4.96 \mathrm{~m} \AA$.

then differences in apparent $\mathrm{CH}$ and $\mathrm{CN}$ line widths would be expected. Alternatively, part of the $\mathrm{CH}$ could be formed in a shockheated region along the line of sight toward $\zeta$ Oph. Such regions have been invoked to explain the observed abundances of $\mathrm{CH}^{+}$in diffuse clouds (Elitzur and Watson 1980). In particular, the shock model of Draine (1986) of the $\zeta$ Oph cloud has $\sim 20 \%$ of the $\mathrm{CH}$ molecules located at the LSR velocity of 2.85 $\mathrm{km} \mathrm{s}^{-1}$. At low resolution, this component should cause a broadening of the $\mathrm{CH}$ profile. $\mathrm{CO}$ and $\mathrm{CN}$ are not expected to be formed efficiently in high-temperature regions, consistent with the fact that no $\mathrm{CO}$ was detected at millimeter wavelengths at $V_{\mathrm{LSR}} \approx 2-5 \mathrm{~km} \mathrm{~s}^{-1}$ (Langer et al. 1987).

Theoretical profiles of the $\mathrm{CH} A^{2} \Delta-X^{2} \Pi(0,0) 4300 \AA$ feature are shown in Figure 3 at $\lambda / \Delta \lambda=8 \times 10^{4}$ and $4.3 \times 10^{5}$. If model 3 is realistic, observations at the higher resolution should reveal not only the $\Lambda$-doubling but also an asymmetry in the profile caused by the complex velocity structure. The comparison between observed (see Danks, Federman, and Lambert 1984) and calculated $\mathrm{CH} 4300 \AA$ lines is affected by uncertainties in the ratio of oscillator strengths $f_{00}^{B X} / f_{00}^{A X}$. If, for 

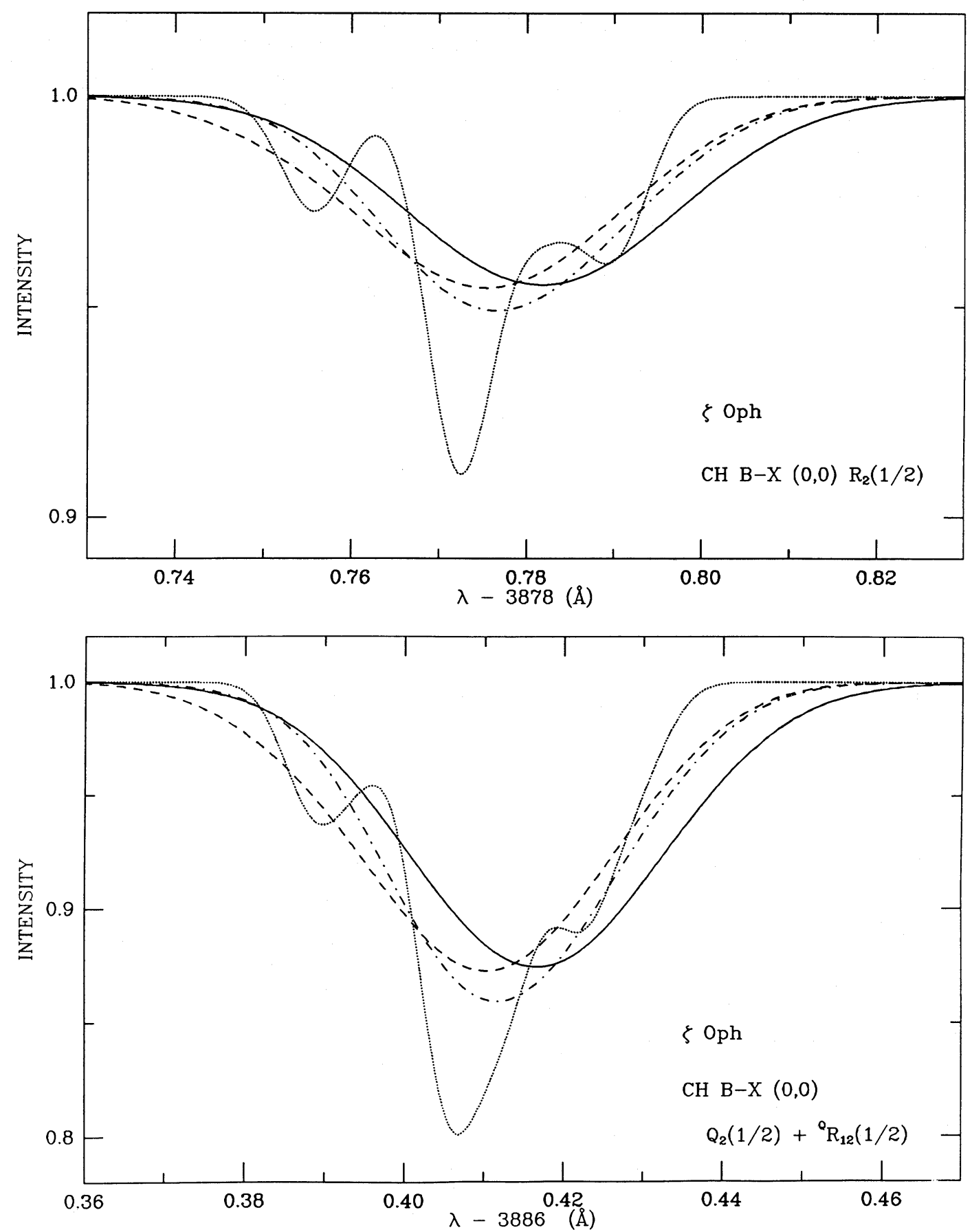

FIG. 2. - Top: synthetic spectra of the CH $B-X(0,0) R_{2}(1 / 2)$ interstellar absorption feature toward $\zeta$ Oph. Solid curve (model 1$)$ is computed for a single velocity component with $b=1.2 \mathrm{~km} \mathrm{~s}^{-1}$. Dashed (model 3) and dot-dash (model 2) curves are computed for four closely spaced velocity components, each of which has $b=0.353 \mathrm{~km} \mathrm{~s}^{-1}$. The resolution is $24.8 \mathrm{~m} \AA$ for all three. Dotted curve is model 3 at $4.96 \mathrm{~m} \AA$ resolution. Bottom: synthetic spectra, as in top panel, but for the CH $B-X(0,0) Q_{2}(1)+{ }^{Q} R_{12}(1)$ interstellar absorption feature toward $\zeta$ Oph. The separation of unresolved line components is $0.488 \mathrm{~km} \mathrm{~s}{ }^{-1}(6.33 \mathrm{~m} \AA)$, as indicated in the text. The previously adopted separation of Lien (1984) that was based on the term energies of Botterud et al. (1973) is rather larger: $1.28 \mathrm{~km} \mathrm{~s}{ }^{-1}(16.6 \mathrm{~m} \AA)$.

example, the oscillator strength adopted by Lien (1984), $f_{00}^{A X}=$ $5.3 \times 10^{-3}$, is used in place of the $5 \%$ smaller value in Table 2 , then the calculated equivalent widths for the $4300 \AA$ feature in Table 3 become $W_{\lambda}=18.19,17.29$, and $17.57 \mathrm{~m} \AA$ for models 1 , 2 , and 3 , respectively. This difference is well within the uncertainty in the oscillator strengths and the higher oscillator strength improves the agreement with observation. Indeed, this point can be carried further to establish that the values of the column densities of interstellar $\mathrm{CH}$ and $\mathrm{CN}$ toward $\zeta \mathrm{Oph}$ are now limited principally by uncertainties in oscillator strengths. The accurate reproduction of well-observed line strengths and profiles for two quite different line-broadening mechanisms with essentially equal column densities indicates that the small saturation effects have been fully included in the analysis of these relatively weak lines. With the assignment of $1 \%-$ $2 \%$ uncertainties to the observed equivalent widths for $B-X$ 


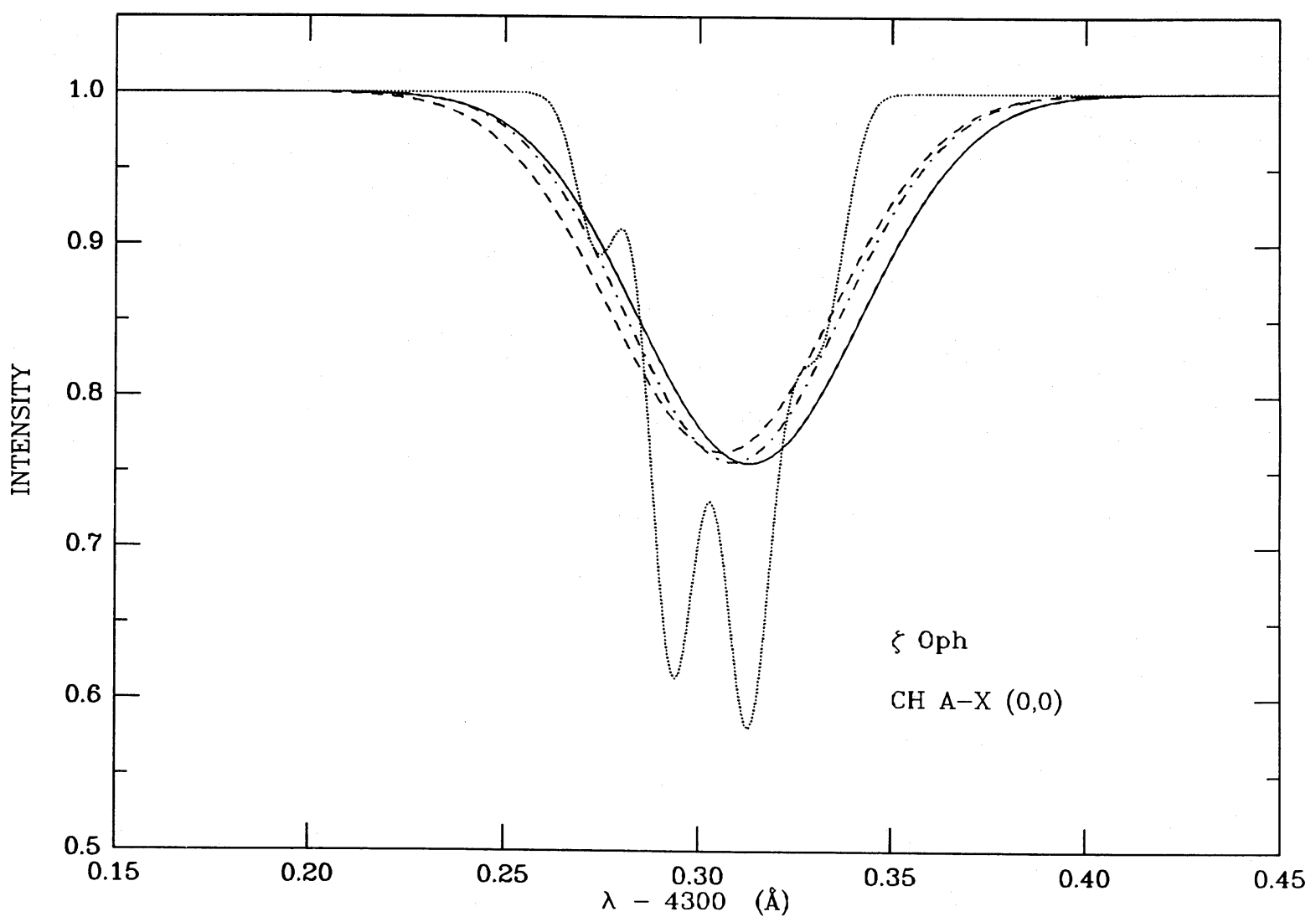

FIG. 3.-Theoretical spectra of the $\mathrm{CH} A-X(0,0)$ interstellar absorption feature toward $\zeta$ Oph. The three shallow and smooth curves were computed for a resolving power $\lambda / \Delta \lambda=8 \times 10^{4}$. Solid curve is for model 1 , the dot-dash curve is for model 2 , and the dashed curve is for model 3 . Dotted curve shows the appearance of the same absorption at higher resolution, $\lambda / \Delta \lambda=4.3 \times 10^{5}$.

lines, $3 \%$ uncertainty to the line broadening, and $10 \%$ uncertainty to the oscillator strengths, the recommended column densities are $N(\mathrm{CH})=(2.51 \pm 0.26) \times 10^{13} \mathrm{~cm}^{-2}$ and $N(\mathrm{CN})=(2.91 \pm 0.30) \times 10^{12} \mathrm{~cm}^{-2}$.

The main conclusion to be drawn from this analysis is that the best existing optical absorption line data are comparably well interpreted by pure thermal broadening at $T \approx 1200 \mathrm{~K}$ and by a combination of turbulence and low-temperature thermal broadening. The radio emission-line data (Langer, Glassgold, and Wilson 1987) and various diagnostic probes of temperature (van Dishoeck and Black 1986a, b) favor the latter explanation. The issue of the nature of the line broadening toward $\zeta$ Oph, as a purely observational problem, can be solved only by further optical spectroscopic measurements at much higher resolution.

\section{CO ABSORPTION LINES}

The interstellar CO molecule is exceptional in that it can be observed by means of absorption lines in the ultraviolet and infrared and by emission lines at millimeter wavelengths (van Dishoeck and Black 1987). Because radio line data on CO have been used above to investigate some effects of velocity structure on the interpretation of absorption lines of other species, it seems worthwhile to consider the interpretation of CO absorption lines in diffuse clouds, too.

Most ultraviolet bands of $\mathrm{CO}$ have a very compact line structure that cannot be resolved in data provided by the Copernicus spectrometer $(\lambda / \Delta \lambda \approx 20,000-30,000)$ and by the International Ultraviolet Explorer $(\lambda / \Delta \lambda \approx 13,000)$. For all but the weakest $\mathrm{CO}$ absorption features, then, the combined effects of saturation and unresolved line structure severely restrict the accuracy of column densities derived from ultraviolet absorption line data (see Black 1985, and references therein). The analysis of such unresolved blends tends to be quite sensitive to any additional unresolved velocity structure and to the values of $b$ in discrete components.

The same line broadening models of $\S$ III can be used to illustrate the problem of $\mathrm{CO}$ line formation in diffuse clouds like that toward $\zeta$ Oph. The $\operatorname{CO} A^{1} \Pi-X^{1} \Sigma^{+}(1,0)$ band is taken as an example. The adopted line positions and oscillator strengths are collected in Table 4. The line positions are measured values of Simmons, Bass, and Tilford (1969) and are probably accurate to $\pm 0.25 \mathrm{~km} \mathrm{~s}^{-1}$. The oscillator strengths are based on the measured lifetimes and inferred transition moment function of Field et al. (1983) as described by van Dishoeck and Black $(1986 b)$. The inverse lifetime is $A=9.57 \times 10^{7} \mathrm{~s}^{-1}$. Fractional level populations, $\eta$, presume a uniform excitation temperature $T_{\mathrm{ex}}=4 \mathrm{~K}$, consistent with the results of Wannier, Penzias, and Jenkins (1982). When adjusted for the improved oscillator strengths (van Dishoeck and Black $1986 \mathrm{~b}$ ), the data of Wannier, Penzias, and Jenkins indicate $N(\mathrm{CO})=2.0 \times 10^{15} \mathrm{~cm}^{-2}$. As described above (eq. [10]), we adopt $T_{\mathrm{D}}=50 \mathrm{~K}$ and $\Delta V_{t}=0.4095 \mathrm{~km} \mathrm{~s}^{-1}$ for each of the velocity components in model 2 .

Figure 4 shows the effect of instrumental resolution on the appearance of the $A-X(1,0)$ band of $\mathrm{CO}$ toward $\zeta$ Oph in model 2. The line structure of the band does not even appear until $\lambda / \Delta \lambda>10,000$, and the complex velocity structure begins 
TABLE 4

AdOPTED Parameters For the CO $A{ }^{1} \Pi-X^{1} \Sigma^{+}(1,0)$ BAND

\begin{tabular}{ccccccrr}
\hline \hline$J^{\prime \prime}$ & $R\left(J^{\prime \prime}\right)$ & $f$ & $Q\left(J^{\prime \prime}\right)$ & $f$ & $P\left(J^{\prime \prime}\right)$ & $f$ & $\eta$ \\
\hline $0 \ldots \ldots \ldots$ & $1509.749^{\mathrm{a}}$ & $3.430(-2)$ & $\ldots$ & $\ldots$ & $\ldots$ & $\ldots$ & $5.455(-1)$ \\
$1 \ldots \ldots \ldots \ldots$ & 1509.697 & $1.715(-2)$ & 1509.838 & $1.715(-2)$ & $\ldots$ & $\ldots .105(-1)$ \\
$2 \ldots \ldots \ldots \ldots$ & 1509.659 & $1.372(-2)$ & 1509.869 & $1.715(-2)$ & 1510.013 & $3.429(-3)$ & $4.303(-2)$ \\
$3 \ldots \ldots \ldots \ldots$ & 1509.647 & $1.225(-2)$ & 1509.927 & $1.715(-2)$ & 1510.137 & $4.898(-3)$ & $9.507(-4)$ \\
$4 \ldots \ldots \ldots \ldots$ & 1509.648 & $1.143(-2)$ & 1509.996 & $1.715(-2)$ & 1510.280 & $5.714(-3)$ & $4.839(-6)$ \\
$5 \ldots \ldots \ldots \ldots$ & 1509.658 & $1.091(-2)$ & 1510.083 & $1.715(-2)$ & 1510.439 & $6.233(-3)$ & $5.875(-9)$ \\
\hline
\end{tabular}

a Vacuum wavelength in $\AA$.
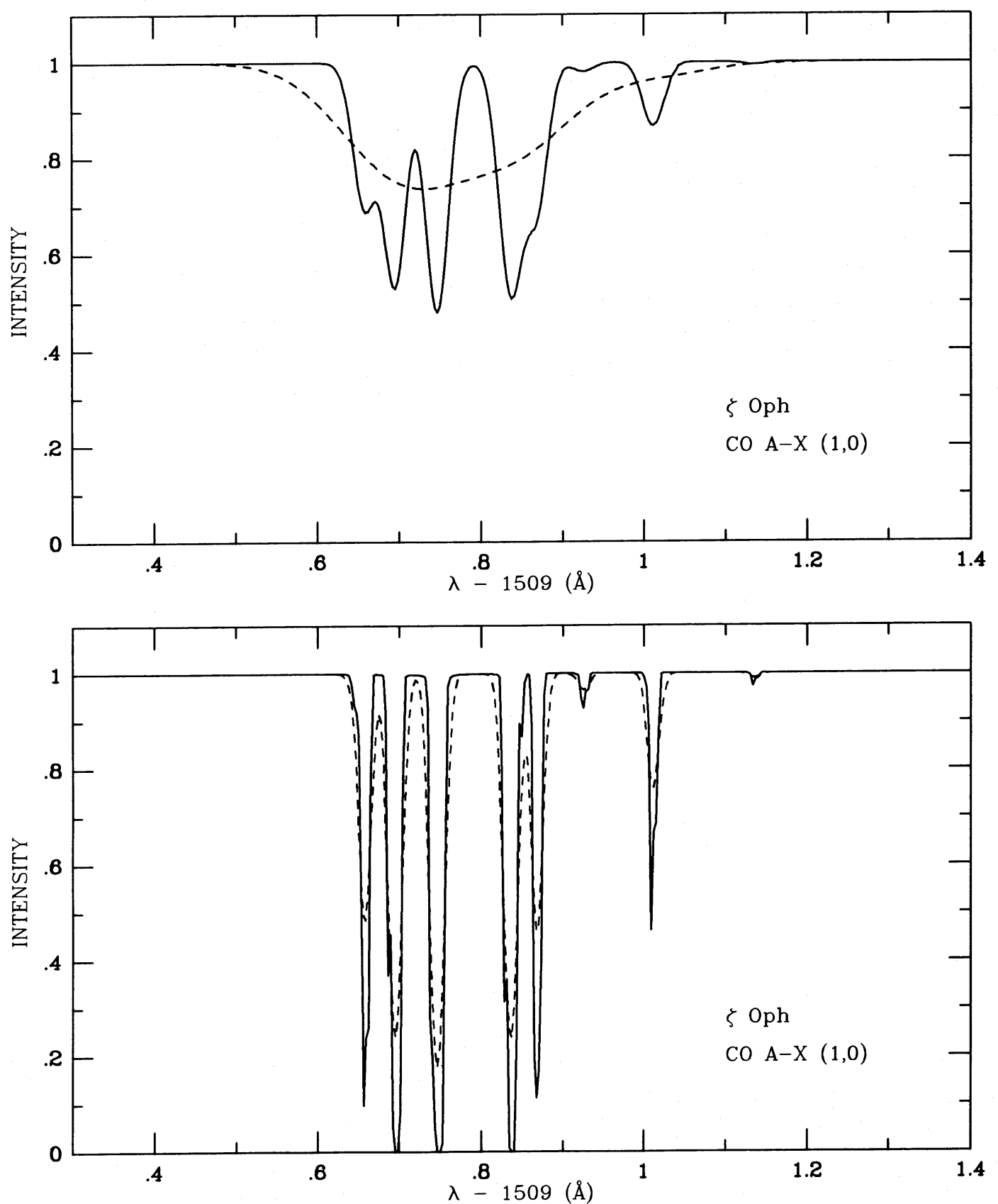

FIG. 4.-Theoretical profiles of the $\mathrm{CO} A-X(1,0)$ band expected for $\zeta$ Oph for velocity model 2 . The total CO column density is $2 \times 10^{15} \mathrm{~cm}^{-2}$. An excitation

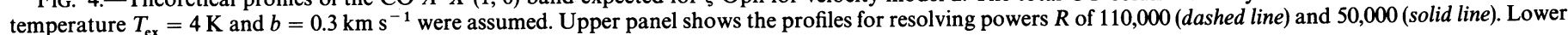
panel shows profiles for $R=100,000$ (dashed line) and 500,000 (solid line), respectively. 
to add discernible shoulders to the line features only for $\lambda / \Delta \lambda \geq 10^{5}$.

At low resolution, of course, only the equivalent width for the entire band can be measured directly. Band profiles have been computed for many values of total column density and their equivalent widths have been determined in order to construct effective curves of growth. Figure 5 presents such curves of growth for both model 1 and model 2 (the latter with several different values of $b$, as well). Also shown are curves of growth for the "band approximation" in which all the population is in a single level and all of the absorption occurs in a single line with an oscillator strength equal to the band value. It is clear from the figure that large errors can be made in derived column densities if the "band approximation" is used or if the velocity structure of the absorbing region is not fully understood. For example, for the $\zeta$ Oph cloud, the observed equivalent width of the $C O A-X(1,0)$ band is $57 \pm 5 \mathrm{~m} \AA$. With model 2 , the column density inferred from this one band is $(7.0 \pm 2.0) \times 10^{14} \mathrm{~cm}^{-2}$, while with model 1 it is $(5.2 \pm 1.3) \times 10^{14} \mathrm{~cm}^{-2}$. The band approximation, however, yields a column density of $7 \times 10^{14} \mathrm{~cm}^{-2}$ only if the Doppler parameter is quite large, $b=3.1 \mathrm{~km} \mathrm{~s}^{-1}$. The $\mathrm{CO}$ column density $(7 \pm 2) \times 10^{14} \mathrm{~cm}^{-2}$ deduced from this one band is significantly smaller than the column density $(2.0 \pm 0.3) \times 10^{15} \mathrm{~cm}^{-2}$ derived from all the ultraviolet data, with the greatest weight on the weakest bands of the $A-X$ system, as mentioned above. This might indicate that the uncertainties in the $(1,0)$ equivalent width have been underestimated. Another example is provided by the line of sight toward
HD 26571. The observed equivalent width of the CO $A-X(1$, $0)$ band is $(147 \pm 10) \mathrm{m} \AA$ (Joseph et al. 1986), which implies $N(\mathrm{CO})=1.2 \times 10^{17} \quad \mathrm{~cm}^{-2}$ at $b=5 \quad \mathrm{~km}^{-1}$ and $N(\mathrm{CO})=1.4 \times 10^{18} \mathrm{~cm}^{-2}$ at $b=1 \mathrm{~km} \mathrm{~s}^{-1}$, in the band approximation. If the line structure is taken into account, however, the observed equivalent width gives $N(\mathrm{CO})=2.2 \times 10^{16} \mathrm{~cm}^{-2}$ for $b=1 \mathrm{~km} \mathrm{~s}^{-1}$ and $T_{\mathrm{ex}}=4 \mathrm{~K}$, which is almost two orders of magnitude less than the result for the band approximation. It is important to note that the effective curves of growth for model 2 presented in Figure 5 apply only to the $(1,0)$ band and that the corresponding curves for other bands may differ in shape and absolute placement. Therefore, it is not guaranteed that an empirical curve of growth can ever be constructed from measurements of several different $\mathrm{CO}$ bands without a detailed model analysis for each band of the sort described here. The best solution, of course, will lie in future ultraviolet observations at sufficiently high resolution to provide measurements of individual lines. The highest resolution mode of the high-resolution spectrograph on the Hubble Space Telescope $\left(\lambda / \Delta \lambda \approx 10^{5}\right)$ will be adequate to reveal line structure, but not necessarily to sort out any complicated velocity structure.

An alternative approach to determining $\mathrm{CO}$ abundances in diffuse clouds will be afforded by absorption measurements in the $v=1-0$ vibration-rotation lines near $4.6 \mu \mathrm{m}$ wavelength. Not only is the rotational line splitting relatively much larger, but the lines in diffuse clouds will typically be weak enough that saturation effects will be small. In order to demonstrate the potential value of this technique, we show the expected

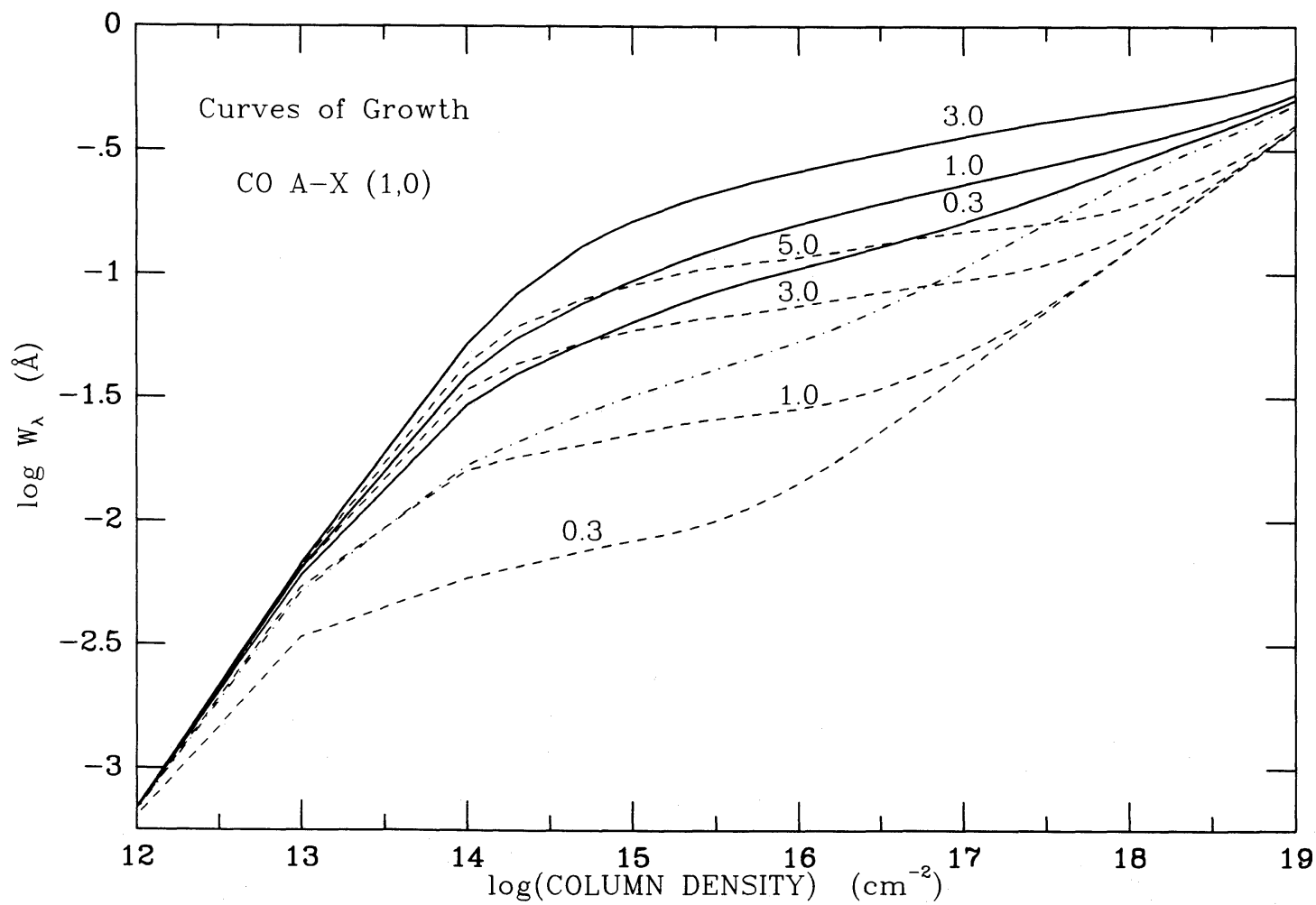

FIG. 5.-Effective curves of growth for the $\mathrm{CO} A-X(1,0)$ band. The equivalent widths have been computed by direct integration over the theoretical profiles. The

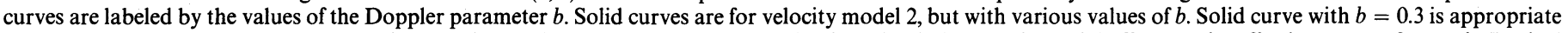

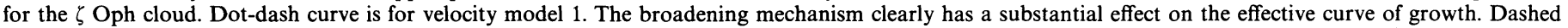
curves are for the "band approximation" in which the absorption is assumed to take place in a single feature without rotational fine structure. 

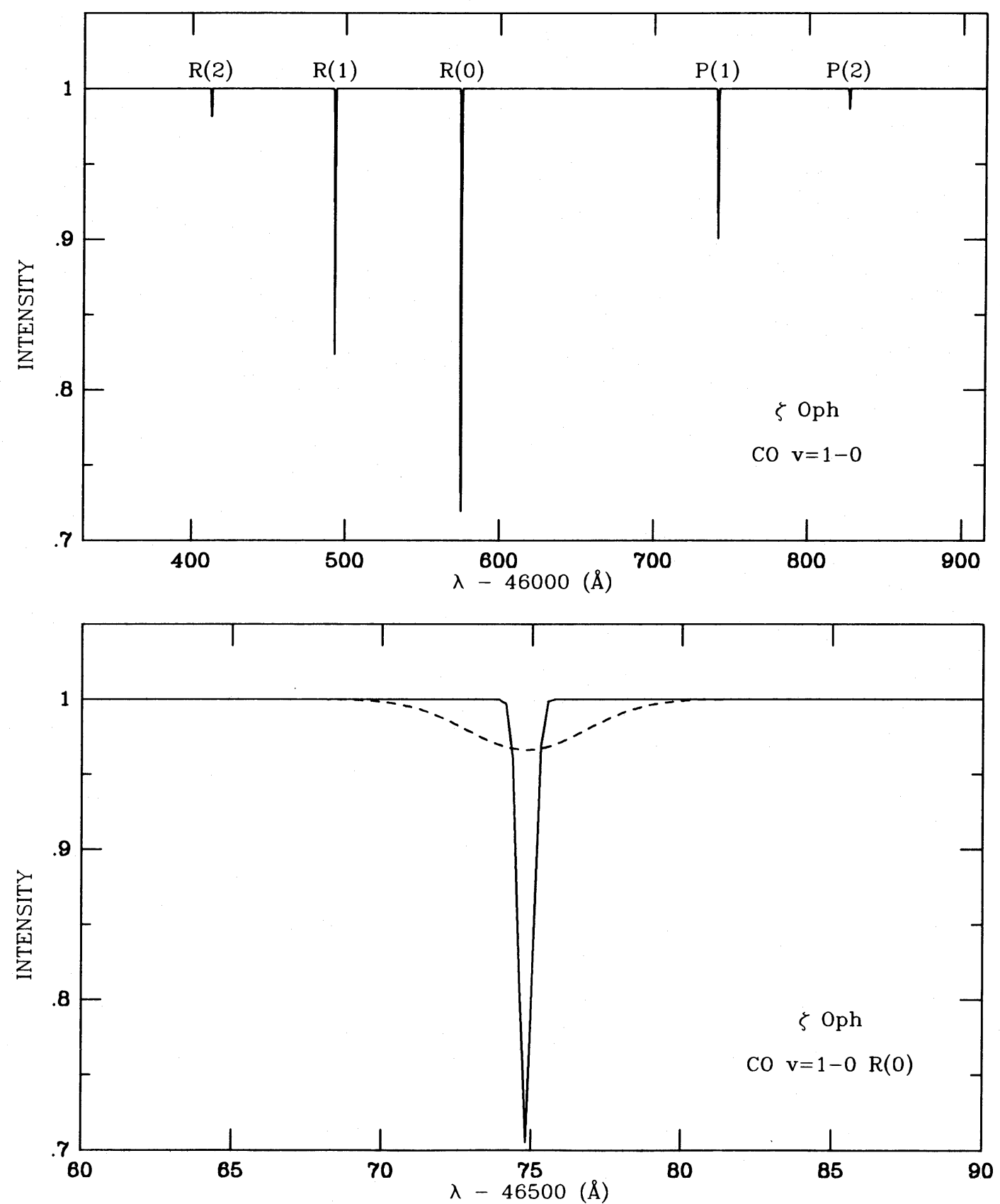

Fig. 6.-Top: the predicted spectrum of the $v=1 \leftarrow 0$ vibration-rotation band of interstellar CO around $4.6 \mu \mathrm{m}$ in $\zeta$ Oph at a resolving power $\lambda / \Delta \lambda=10^{5}$. Bottom: detail of the profile of the $R(0)$ line at resolving power of $\lambda / \Delta \lambda=10^{5}$ (solid line) and $\lambda / \Delta \lambda=10^{4}$ (dashed line).

appearance of the strongest lines of the $\mathrm{CO} v=1-0$ band and of the profile of the $R(0)$ line for $\zeta$ Oph in Figure 6. The adopted line positions (Guelachvili 1979) and oscillator strengths (Chackerian and Tipping 1983) are collected in Table 5. The inverse lifetime of the $v=1$ levels is $A=36.1 \mathrm{~s}^{-1}$. Note that the $R(0)$ line in the $\zeta$ Oph spectrum is expected to have a central depth $30 \%$ of the continuum level at $\lambda / \Delta \lambda=10^{5}$ but only $3 \%$ at $\lambda / \Delta \lambda=10^{4}$ (Fig. $6 b$ ). The higher sensitivity usually expected at the lower resolution will not necessarily make such unresolved infrared lines measureable at $\lambda / \Delta \lambda \approx 10^{4}$ unless weak, narrow, telluric absorptions can be removed at a level of much better than $1 \%$.
TABLE 5

Adopted Parameters for CO $v=1 \leftarrow 0$ Vibration-Rotation Band

\begin{tabular}{ccccc}
\hline \hline$J^{\prime \prime}$ & $R\left(J^{\prime \prime}\right)$ & $f$ & $P\left(J^{\prime \prime}\right)$ & $f$ \\
\hline $0 \ldots \ldots \ldots \ldots$ & $2147.08158^{\mathrm{a}}$ & $1.186(-5)$ & $\ldots$ & $\ldots$ \\
$1 \ldots \ldots \ldots \ldots$ & 2150.85646 & $7.925(-6)$ & 2139.42653 & $3.939(-6)$ \\
$2 \ldots \ldots \ldots \ldots$ & 2154.59603 & $7.147(-6)$ & 2135.54663 & $4.717(-6)$ \\
$3 \ldots \ldots \ldots \ldots$ & 2158.30016 & $6.820(-6)$ & 2131.63203 & $5.045(-6)$ \\
$4 \ldots \ldots \ldots \ldots$ & 2161.96869 & $6.643(-6)$ & 2127.68286 & $5.221(-6)$ \\
$5 \ldots \ldots \ldots \ldots$ & 2165.60149 & $6.535(-6)$ & 2123.69927 & $5.329(-6)$ \\
\hline
\end{tabular}

a Vacuum wavenumber in $\mathrm{cm}^{-1}$. 
V. DISCUSSION

In $\S \S$ III and IV we showed that small-scale velocity structure is important, not only for abundance determinations of species such as $\mathrm{CH}, \mathrm{CN}$, and $\mathrm{CO}$, but also for understanding broadening mechanisms that reflect the very physical nature of the absorbing gas. However, the small-scale velocity structure, even if present, does not always have directly observable consequences. Consider the strongly saturated interstellar $\mathrm{C}$ II line in $\zeta$ Oph at $\lambda=1334.5323 \AA\left(f=0.129, A=2.9 \times 10^{8} \mathrm{~s}^{-1}\right)$ for which $W_{\lambda}=189 \mathrm{~m} \AA$ (Morton 1975). As mentioned in $\S$ I, Crutcher (1975) identified five distinct velocity components in $\mathrm{Na}$, the central and strongest one of which at $V_{\mathrm{LSR}}=-0.3 \mathrm{~km} \mathrm{~s}^{-1}$ can be said to contain all of the finer structure of our models 2 and 3. We have computed effective curves of growth by direct integration of line profiles for this C II feature using Crutcher's (1975) velocity distribution and $b$-values both with and without the finer structure in the strongest component. There is no significiant difference in observable profiles and equivalent widths of the features with and without the finer structure when the column density $N\left(\mathrm{C}^{+}\right) \geq 10^{16} \mathrm{~cm}^{-2}$. This is not surprising, because once the core of the line is fully saturated, unresolved structure within the core can have no appreciable effect on the total equivalent width or the shape of the wings. There is, of course, a significant difference in the line profile computed with Crutcher's velocity distribution and the profile computed with a single-component curve of growth like the one with $b=6.5 \pm 0.5 \mathrm{~km} \mathrm{~s}^{-1}$ used by Morton (1975) to analyze the measured equivalent width. Morton inferred a column density $N\left(\mathrm{C}^{+}\right)=(0.6-1.4) \times 10^{17} \mathrm{~cm}^{-2}$ using the empirical curve of growth, while we find $N\left(\mathrm{C}^{+}\right)=2.6 \times 10^{17}$ $\mathrm{cm}^{-2}$ based on the eight-component profile. An accurate determination of the $\mathrm{C}^{+}$column density must await measurements of the intrinsically weak C II] intersystem lines near $2325 \AA$ wavelength (Hobbs, York, and Oegerle 1982), for which the accuracy might eventually be limited primarily by uncertainties in the oscillator strengths (Cowan, Hobbs, and York 1982; Nussbaumer and Storey 1981).

In the preceding sections, we have seen examples of situations in which unresolved velocity structure is further complicated by unresolved structure in the atomic or molecular transitions themselves. In the case of the $\mathrm{CH} B-X(0,0)$ lines, intrinsic splittings of the order of $1 \mathrm{~km} \mathrm{~s}^{-1}$ occur and the recent improvements in the basic spectroscopic data have been very important (Bernath 1987a, b). There are other kinds of intrinsic structure that will affect detailed interpretation of interstellar absorption line data. Isotope shifts in the spectra of light atoms like carbon are also of the order of $1 \mathrm{~km} \mathrm{~s}^{-1}$ in relative velocity. For example, the separation of the ${ }^{12} \mathrm{C}$ and ${ }^{13} \mathrm{C}$ components of the $\mathrm{C}$ II $1334.5 \AA$ resonance line is expected to be $\lambda_{12}-\lambda_{13}=0.0153 \AA$ or $\Delta V=3.4 \mathrm{~km} \mathrm{~s}^{-1}$ (Clark 1983, 1984; Bernheim and Kittrell 1980). This structure will have effects of the order of a few percent on a single-component curve of growth if the isotope abundance ratio is as large as $\left[{ }^{12} \mathrm{C}\right] /\left[{ }^{13} \mathrm{C}\right]=43$ (Hawkins and Jura 1987). Such a small relative abundance will probably cause the rarer species to be unobservable except in regions of very low column density where the strong resonance lines might not be overwhelmingly saturated. In thicker interstellar clouds, the isotopic species ${ }^{13} \mathrm{C}$ and ${ }^{13} \mathrm{C}^{+}$may be more easily observable by their finestructure emission lines at $370 \mu \mathrm{m}$ and $157 \mu \mathrm{m}$, respectively, which are shifted by $50-80 \mathrm{~km} \mathrm{~s}^{-1}$ from those of the main species (Cooksy et al. 1986; Cooksy, Blake, and Saykally 1986).
In magnesium, the isotope abundances are much closer: the mass 24,25 , and 26 varieties comprise $\sim 79 \%, 10 \%$, and $11 \%$, respectively, of the terrestrial magnesium. As pointed out by Bernath, Black, and Brault (1985), the $\mathrm{Mg}$ I] $3 s^{2}{ }^{1} S_{0}-3 s 3 p{ }^{3} P_{1}$ transition at $\lambda=4571.104 \AA$ is potentially observable in interstellar absorption. The ${ }^{26} \mathrm{Mg}-{ }^{24} \mathrm{Mg}$ isotope shift in the transition is $0.61 \pm 0.01 \mathrm{~km} \mathrm{~s}^{-1}$ (Veseth 1987). Figure 7 shows predicted profiles of the $\mathrm{Mg}$ I] $4571.1 \AA$ feature appropriate for the line of sight toward HD 169454. The HD 169454 cloud has a single narrow velocity component with $b=0.43 \mathrm{~km} \mathrm{~s}^{-1}$ based on millimeter wavelength $\mathrm{CO}$ observations toward the star (Jannuzi et al. 1988). The adopted column density of neutral magnesium, $N(\mathrm{Mg})=6.8 \times 10^{14} \mathrm{~cm}^{-2}$, is based upon a model of the chemistry and ionization of the cloud (van Dishoeck and Black 1988) in which the total hydrogen column density $N(\mathrm{H})+2 N\left(\mathrm{H}_{2}\right)=3.6 \times 10^{21} \mathrm{~cm}^{-2}$, the gas phase magnesium abundance is $[\mathrm{Mg} / \mathrm{H}]=5.1 \times 10^{-6}$, and the effects of large molecules on the atomic ionization balance have been taken into account (Lepp et al. 1988). The solid, short-dash, and long-dash curves are computed for resolving powers of $\lambda / \Delta \lambda=10^{7}, 10^{6}$, and $2 \times 10^{5}$, respectively. The structure in the absorption line profile due to the ${ }^{24} \mathrm{Mg}-{ }^{26} \mathrm{Mg}$ isotope splitting appears only as a slight asymmetry.

Hyperfine splitting is also a potential source of small-scale profile structure in some spectra. One example of observable hyperfine structure is provided by $\mathrm{Mn}$ I. The strongest resonance line of $\mathrm{Mn} \mathrm{I}, 4 s^{2}{ }^{6} S_{5 / 2}-4 s 4 p^{6} P_{7 / 2}^{o}$ at $\lambda=4030.753 \AA$ has six hyperfine structure components with splittings ranging from $c \Delta \lambda / \lambda=0.37$ to $1.2 \mathrm{~km} \mathrm{~s}^{-1}$ (Booth, Shallis, and Wells 1983), comparable to the velocity structure in the $\zeta$ Oph cloud as discussed above. At $\lambda / \Delta \lambda=10^{5}$, the combination of hyperfine structure and velocity components in $\zeta$ Oph model 2 obliterates any structure in the calculated interstellar line profile, although it is predicted to be at least $50 \%$ wider than the corresponding profile computed for a single velocity component with no hyperfine structure. On the basis of an extensive ionization model of the $\zeta$ Oph cloud (Lepp et al. 1988), we predict an equivalent width for this interstellar $\mathrm{Mn}$ I feature of $W_{\lambda}=7.6 \mathrm{~m} \AA$ in $\zeta$ Oph. Although this line will be too weak to show significant effects of hyperfine structure on the curve of growth as it does in stellar spectra (Booth and Blackwell 1983), such effects may become important in thicker clouds.

Finally, it is apparent that high-resolution investigations of the broadening of interstellar lines address the question of the nature of the "turbulence" in diffuse clouds. The CO observations towards $\zeta$ Oph of Langer, Glassgold, and Wilson (1987) suggest several distinct condensations separated by $\sim 1 \mathrm{~km} \mathrm{~s}^{-1}$ in radial velocity and characterized by turbulent widths $\Delta V_{t} \approx$ $0.4 \mathrm{~km} \mathrm{~s}^{-1}$. The strongest velocity feature in $\mathrm{CO}$ shows approximately uniform emission over at least $6^{\prime}$, but with a gradient of $1 \mathrm{~km} \mathrm{~s}^{-1} \mathrm{pc}^{-1}$ for an adopted distance of $D=140$ pc. Emission in the weaker components varies on scales of $2^{\prime}$ $\left(\Delta z=2.5 \times 10^{17} \mathrm{~cm}\right)$. To describe the line-of-sight structure, we use model $\mathrm{G}$ of the $\zeta$ Oph cloud (van Dishoeck and Black $1986 b$ ). In this model, $90 \%$ of the total $\mathrm{CH}$ or $\mathrm{CO}$ column density is contained within a region of thickness $\Delta z=2.6 \times 10^{18} \mathrm{~cm}$, in which the mean density is $\left\langle n_{\mathrm{H}}\right\rangle=330$ $\mathrm{cm}^{-3}$. If this region is composed of five clumps with a $50 \%$ line-of-sight filling factor, then the size of each clump is $\Delta z \approx 2.6 \times 10^{17} \mathrm{~cm}$, in excellent agreement with the apparent scale on the sky. In such a scheme, a clump density $n_{\mathrm{H}}=440$ $\mathrm{cm}^{-3}$, a turbulent velocity $V_{t}=\Delta V_{t} \approx 0.4 \mathrm{~km} \mathrm{~s}^{-1}$, and a length scale $\Delta z=2.5 \times 10^{17} \mathrm{~cm}$ imply a local heating input due to 


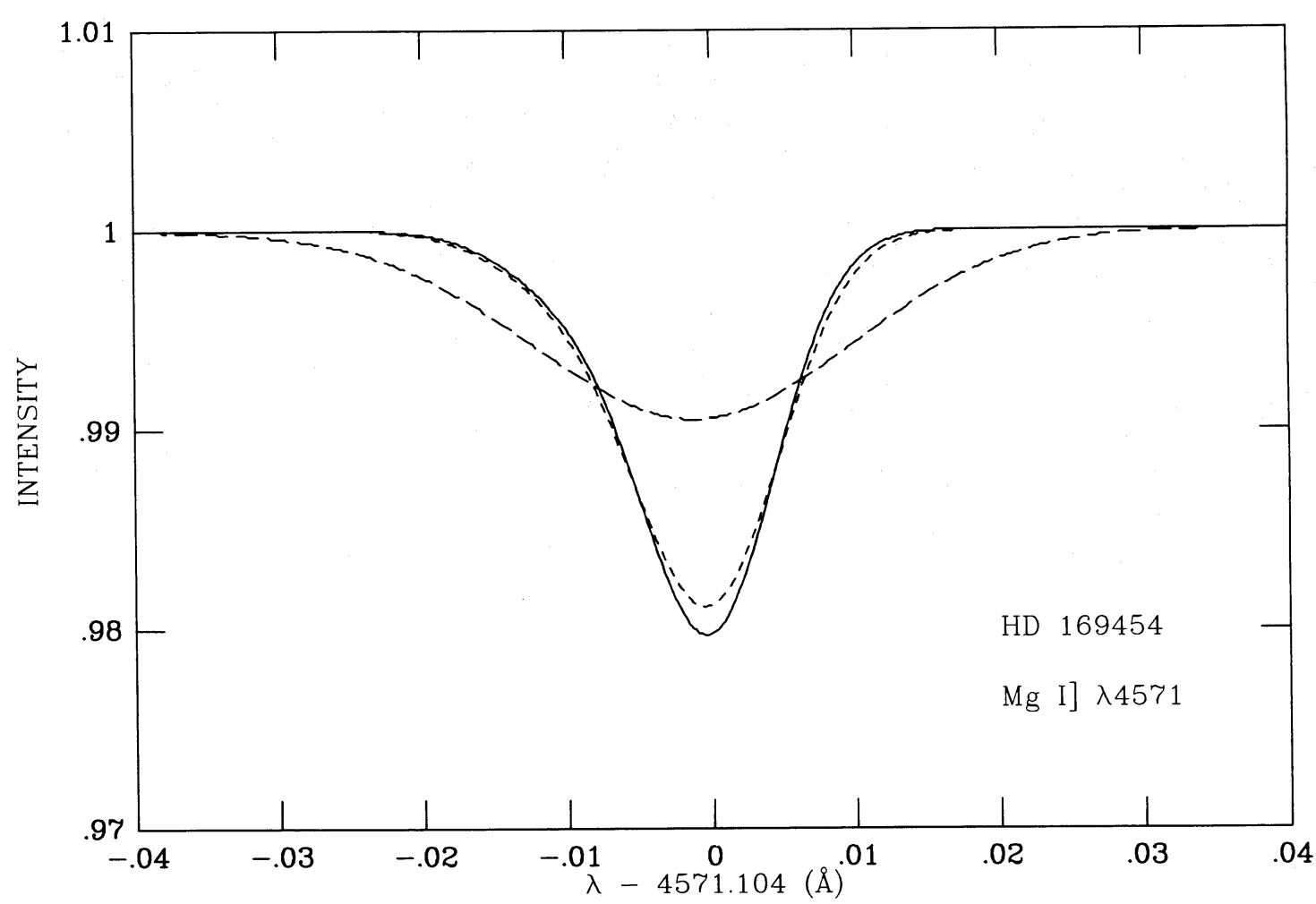

FIG. 7.-Predicted profiles of the $\mathrm{Mg}$ I] $4571.1 \AA$ feature toward HD 169454. Solid short-dash, and long-dash curve are computed for resolving powers of $\lambda / \Delta \lambda=10^{7}, 10^{6}$, and $2 \times 10^{5}$, respectively. The ${ }^{24} \mathrm{Mg}$ and ${ }^{26} \mathrm{Mg}$ isotopic species were taken to have fractional abundances of 0.875 and 0.125 , respectively, and ${ }^{25} \mathrm{Mg}$ was ignored here because its line position has not been measured.

dissipation of turbulence of $\Gamma=1.2 \times 10^{-25} \mathrm{ergs} \mathrm{s}^{-1} \mathrm{~cm}^{-3}$ (see eq. [18] of Black 1987). This is $\sim 100$ times smaller than the total heating and cooling rates for microscopic processes such as photoelectric heating and atomic fine-structure line cooling in the adopted model cloud. This rate of turbulent energy dissipation, however, is equal to the time-averaged, global rate of mechanical energy input to the interstellar medium from supernova remants in the Galaxy as estimated by Cox (1979). Thus we conclude that if the line broadening models 2 and 3 and our chemical model of the $\zeta$ Oph cloud are realistic, then the source of apparent turbulence in this diffuse cloud can be explained. There is, of course, no way of knowing directly at this time whether there is structure on even smaller scales.
It is a pleasure to thank P. Crane, A. Glassgold and W. Langer for sending us preprints of their papers prior to publication and for helpful discussions. We thank P. Bernath for communicating results of his spectroscopic studies of $\mathrm{CH}$ prior to publication. We are grateful to B. T. Draine, E. B. Jenkins, and R. Narayan for useful conversations. This work is supported in part by the National Aeronautics and Space Administration astrophysical theory program through NAGW-763 to the University of Arizona, and by the National Science Foundation through grant RII 86-20342 to Princeton University.

\section{REFERENCES}

Bernath, P. F 1987a, J. Chem. Phys. 86, 4838. $1987 b$, private communication

Bernath, P. F., Black, J. H., and Brault, J. W. 1985, Ap. J., 298, 375.

Bernheim, R. A., and Kittrell, C. 1980, Spectrochim. Acta, 35B, 51.

Black, J. H. 1985, in Molecular Astrophysics: State of the Art and Future Directions, ed. G. H. F. Diercksen, W. F. Huebner, and P. W. Langhoff (Dordrecht: Reidel), p. 215

. 1987, in Interstellar Processes, ed. D. J. Hollenbach and H. A. Thron-

son, Jr. (Dordrecht: Reidel), p. 731.

Blades, J. C., Wynne-Jones, I., and Wayte, R. C. 1980, M.N.R.A.S., 193, 849.

Booth, A. J., and Blackwell, D. E. 1983, M.N.R.A.S., 204, 777 .

Booth, A. J., Shallis, M. J., and Wells, M. 1983, M.N.R.A.S., 205, 191.

Botterud, I., Lofthus, A., and Veseth, L. 1973, Phys. Scripta, 8, 218.

Brazier, C. R., and Brown, J. M. 1984, Canadian J. Phys., 62, 1563.

Brzozowski, J., Bunker, P., Elander, N., and Erman, P. 1976, Ap. J., 207, 414

Chackerian, C., and Tipping, R. H. 1983, J. Molec. Spectrosc., 99, 431.

Clark, C. W. 1983, Optics Letters, 8, 572.

. 1984, Ap. J., 285, 322
Cooksy, A. L., Blake, G. A., and Saykally, R. J. 1986, Ap. J. (Letters), 305, L89. Cooksy, A. L., Saykally, R. J., Brown, J. M., and Evenson, K. 1986, Ap. J., 309, 828.

Cox, D. P. 1979, Ap. J., 234, 863.

Cowan, R. D., Hobbs, L. M., and York, D. G. 1982, Ap. J., 257, 373; 265, 582.

Crane, P., Hegyi, D. J., Mandolesi, N., and Danks, A. C. 1986, Ap. J., 309, 822. Crutcher, R. M. 1975, Ap. J., 200, 625.

Crutcher, 1977, Ap. J.(Letters), 217, L109.

Danks, A. C., Federman, S. R., and Lambert, D. L. 1984, Astr. Ap., 130, 62.

de Boer, K. S. 1979, Ap. J., 229, 132.

. 1981, Ap. J., 244, 848.

Draine, B. T. 1986, Ap. J., 310, 408

Elitzur, M., and Watson, W. D. 1980, Ap. J., 236, 172.

Engleman, R. 1974, J. Molec. Spectrosc., 49, 106.

Field, R. W., Benoist d'Azy, O., Lavollee, M., Lopez-Delgado, R., and Tramer,

A. 1983, J. Chem. Phys., 78, 2838.

Gerö, L. 1941, Zs. Phys., 118, 27.

Guelachvili, G. 1979, J. Molec. Spectrosc., 75, 251. 
Hawkins, I., and Jura, M. 1987, Ap. J., 317, 926.

Hobbs, L. M. 1973, Ap. J.(Letters), 180, L79. .1978a, Ap. J., 222, 491 1978b, Ap.J. Suppl., 38, 129.

Hobbs, L. M., York, D. G., and Oegerle, W. 1982, Ap. J. (Letters), 252, L21. Jannuzi, B. T., Lada, C. J., Black, J. H., and van Dishoeck, E. F. 1988, Ap. J., in II press.

Jenkins, E. B. 1986, Ap. J., 304, 739.

Joseph, C. L., Snow, T. P., Seab, C. G., and Crutcher, R. M. 1986, Ap. J., 309, 771.

Jura, M. and Meyer, D. M. 1985, Ap. J. 294, 238.

Langer, W. D., Glassgold, A. E., and Wilson, R. W. 1987, Ap. J., 322, 450.

Larsson, M., and Siegbahn, P. E. M. 1983, J. Chem. Phys., 79, 2270; 85, 4208 (1986).

Larsson, M., Siegbahn, P. E. M., and Ågren, H. 1983, Ap. J., 272, 369.

Lepp, S., Dalgarno, A., van Dishoeck, E. F., and Black, J. H. 1988, Ap. J., 329, in press.
Lien, D. J. 1984, Ap. J., 284, 578.

Liszt, H. S. 1979, Ap. J.(Letters), 233, L147.

Morton, D. C. 1975, Ap. J., 197, 85.

Nachman, P., and Hobbs, L. M. 1973, Ap. J., 182, 481.

Nussbaumer, H., and Storey, P. J. 1981, Astr. Ap., 96, 91.

Palazzi, E., Mandolesi, N., and Crane, P. 1988, Ap. J., 326, 905.

Simmons, J. D., Bass, A. M., and Tilford, S. G. 1969, Ap. J., 155, 345.

Snow, T. P., and Meyers, K. A. 1979, Ap. J., 229, 545

van Dishoeck, E. F., and Black, J. H. 1986a, Ap. J., 307, 332.

. 1986b, Ap.J. Suppl., 62, 109.

1987, in Physical Processes in Interstellar Clouds, ed. G. E. Morfill and M. Scholer (Dordrecht: Reidel), p. 241 1988 , in preparation.

Veseth, L. 1987, J.Phys. B, 20, 235

Wannier, P. G., Penzias, A. A., and Jenkins, E. B. 1982, Ap. J., 254, 100.

Wayte, R. C., Wynne-Jones, I., and Blades, J. C. 1978, M.N.R.A.S., 182, 5P.

JoHN H. BlACK : Steward Observatory, University of Arizona, Tucson, AZ 85721

Ewine F. van Dishoeck: Princeton University Observatory, Peyton Hall, Princeton, NJ 08544 